Supporting Information

\title{
Collision Cross Section Conformational Analyses of Bile Acids via Ion Mobility-Mass Spectrometry
}

\author{
James C. Poland, ${ }^{* \dagger}$ Katrina L. Leaptrot, ${ }^{*+}$ Stacy D. Sherrod, ${ }^{\dagger}$ \\ Charles Robb Flynn, ${ }^{\ddagger}$ and John A. McLean ${ }^{*} \dagger$
}

†Center for Innovative Technology, Department of Chemistry, Institute of Chemical Biology, Institute for Integrative Biosystems Research and Education, Vanderbilt-Ingram Cancer Center, Vanderbilt University, Nashville, TN 37235 USA

‡Department of Surgery; Vanderbilt University Medical Center, Nashville, TN 37235 USA

*Corresponding Author Email: john.a.mclean@vanderbilt.edu

\section{Comments on Untargeted Metabolomics Presented in this Work}

In this Supporting Information we include the arrival time distributions of four $\beta$-muricholic acid ion forms (Figure S1a) and comparative arrival time of distributions for chenodeoxycholic acid and ursodeoxycholic acid (Figure S1b). Figure S2 shows three examples of ion mobilitymass spectrometry spectra with potential metastable adduct behavior. Figure $\mathbf{S 3}$ shows the trendlines for three bile acid subclasses. Figure S4 shows how previously unidentified features from a metabolomics sample fall on the bile acid trendline. In addition, we include tables of all measured bile acids and their drift tube collision cross section values measured in nitrogen $\left({ }^{\mathrm{DT}} \mathrm{CCS}_{\mathrm{N} 2}\right)$ in both positive and negative ionization mode (Tables S1-S3). 
a) Arrival Time Distrubution of $\beta-\mathrm{MCA}$

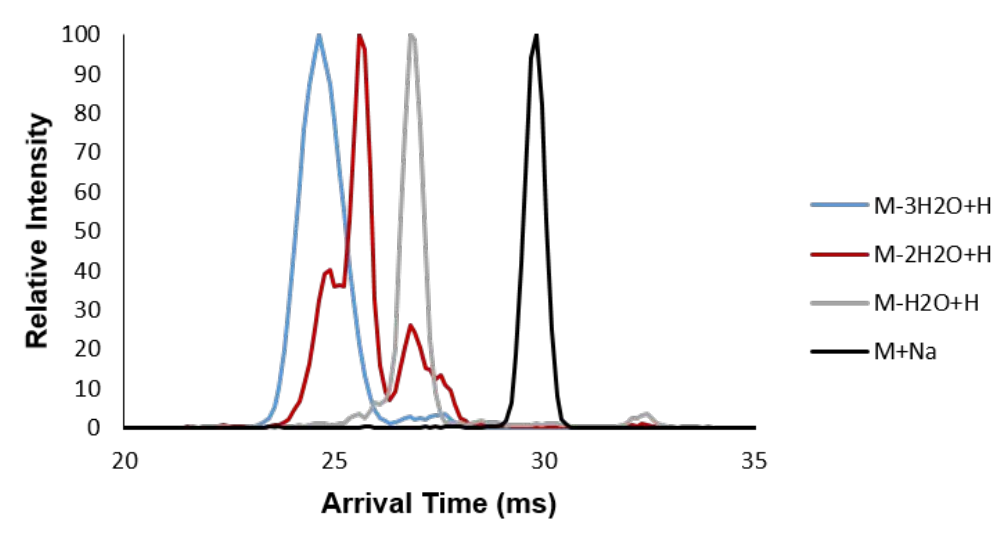

b)

Arrival Time Distribution

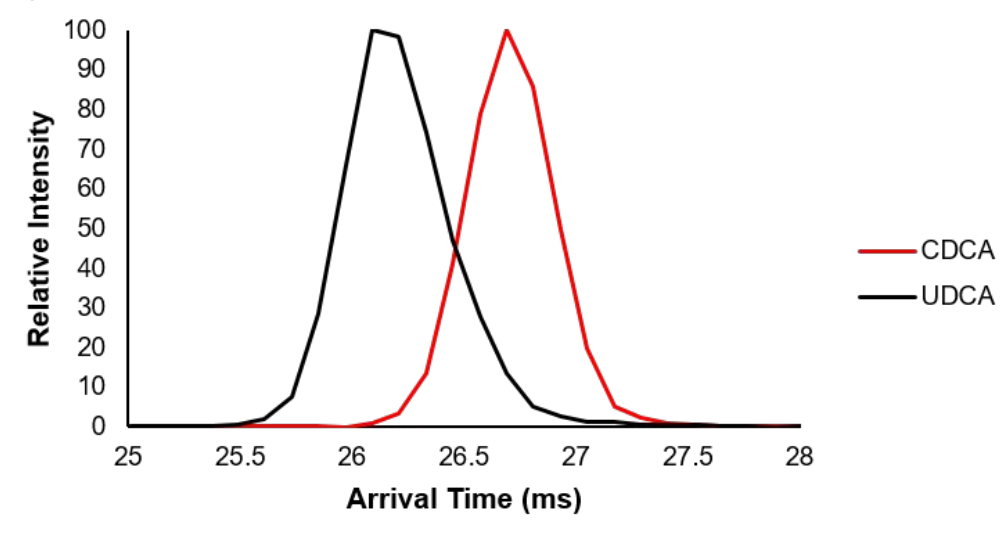

Figure S1. Drift tube ion mobility arrival time distributions (ATD). a) ATDs of the four highest abundance ion forms of $\beta$-muricholic acid, taken in positive ionization mode. b) ATDs of the $[\mathrm{M}-\mathrm{H}]$ ] ion forms of chenodeoxycholic acid and ursodeoxycholic acid, two bile acid isomers, analyzed individually as pure standards. 


\section{Supporting Information}

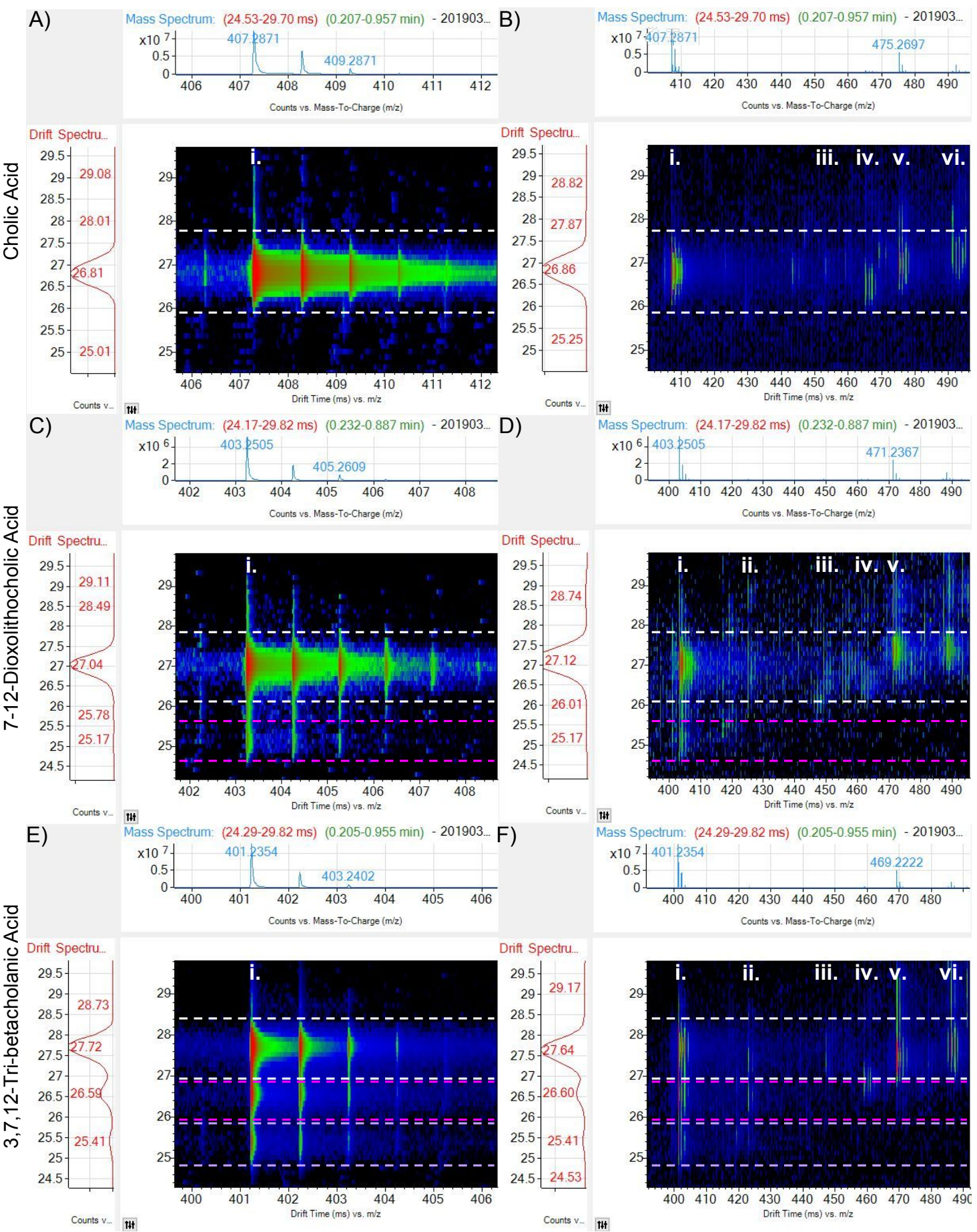

Figure S2. Screenshots from Agilent MassHunter IM-MS Browser showing IM-MS spectra of 3 bile acids analyzed in negative ionization mode. Roman numerals are mass-aligned in each spectra to the observed ion forms: i. $[\mathrm{M}-\mathrm{H}]^{-}$, ii. $[\mathrm{M}+\mathrm{Na}-2 \mathrm{H}]^{-}$, iii. [M+HCOO]-, iv. $[\mathrm{M}+\mathrm{Na}-$ $\mathrm{H}+\mathrm{Cl}]^{-}$, v. [M+Na-H+HCOO$]^{-}$, and vi. [M+K-H+HCOO]- A) Cholic acid had one observed [M-H] ion mobility feature B) which was mobility-aligned with higher $\mathrm{m} / \mathrm{z}$ ion forms (inside the white dashed lines). C) 7-12-Dioxolithocholic acid had two observed [M-H] ion mobility features D) of which the lower mobility feature did not align with higher $\mathrm{m} / \mathrm{z}$ ion forms (inside the pink dashed lines), and the higher mobility feature was mobility-aligned with higher $\mathrm{m} / \mathrm{z}$ ion forms (inside the white dashed lines). E) 3,7,12-Tri-betacholanic acid had three observed $[\mathrm{M}-\mathrm{H}]^{-}$ion mobility features $\mathrm{F}$ ) of which the lowest mobility feature did not align with higher $\mathrm{m} / \mathrm{z}$ ion forms (inside the lavendar dashed lines), the middle mobility feature was mobility-aligned with the lower mobility feature of the [M+Na-2H]- ion form (inside the pink dashed lines), and the highest mobility feature was mobility-aligned with higher $\mathrm{m} / \mathrm{z}$ ion forms (inside the white dashed lines). 


\section{Supporting Information}
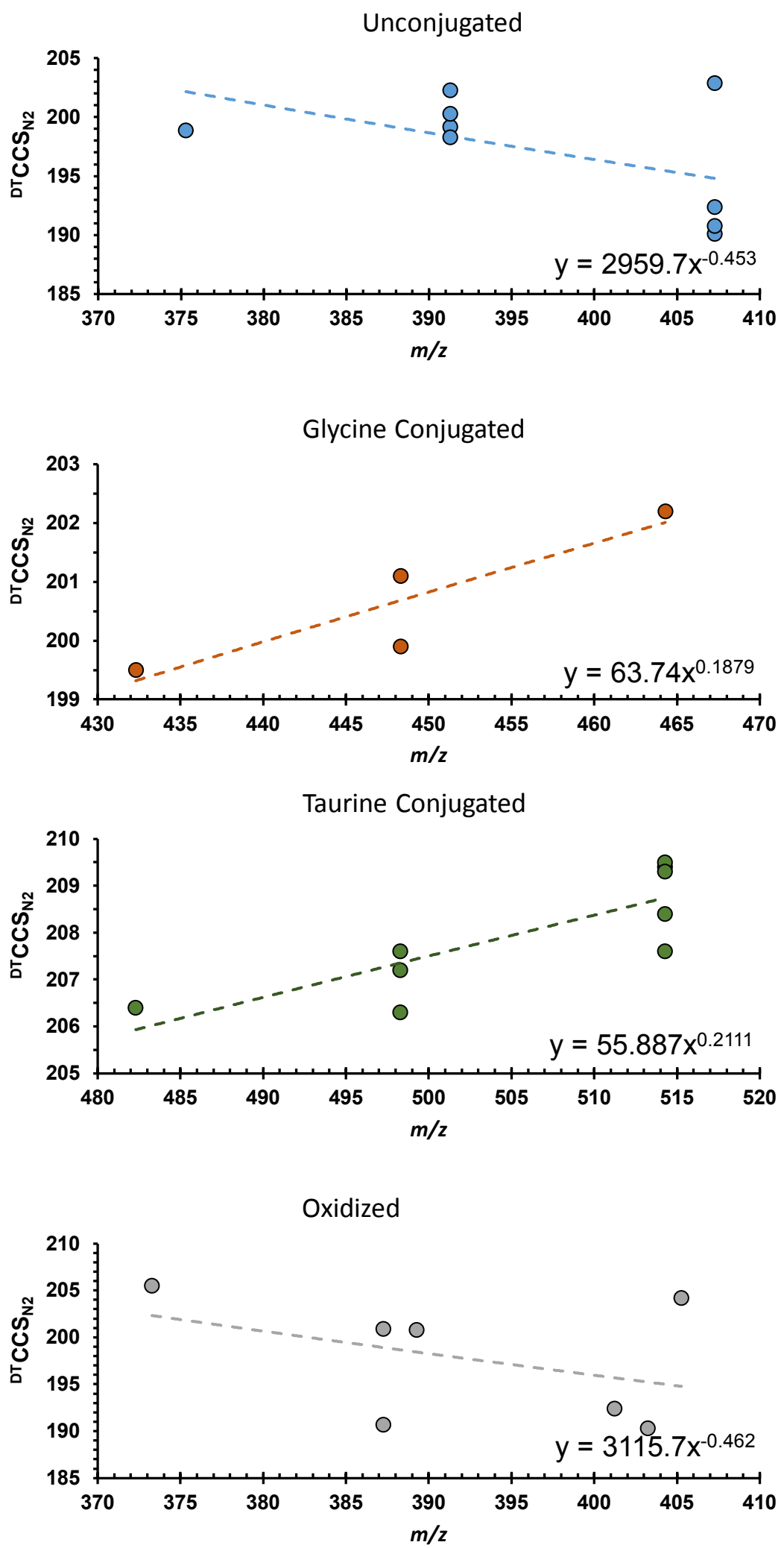

Figure S3. This figure demonstrates the trendlines of the different subclasses. In addition to the general bile acid trendline, the subclasses can be fit to their own individual trendlines, although the sample numbers may not currently be sufficient to accurately represent individual subclass trends. 


\section{Supporting Information}

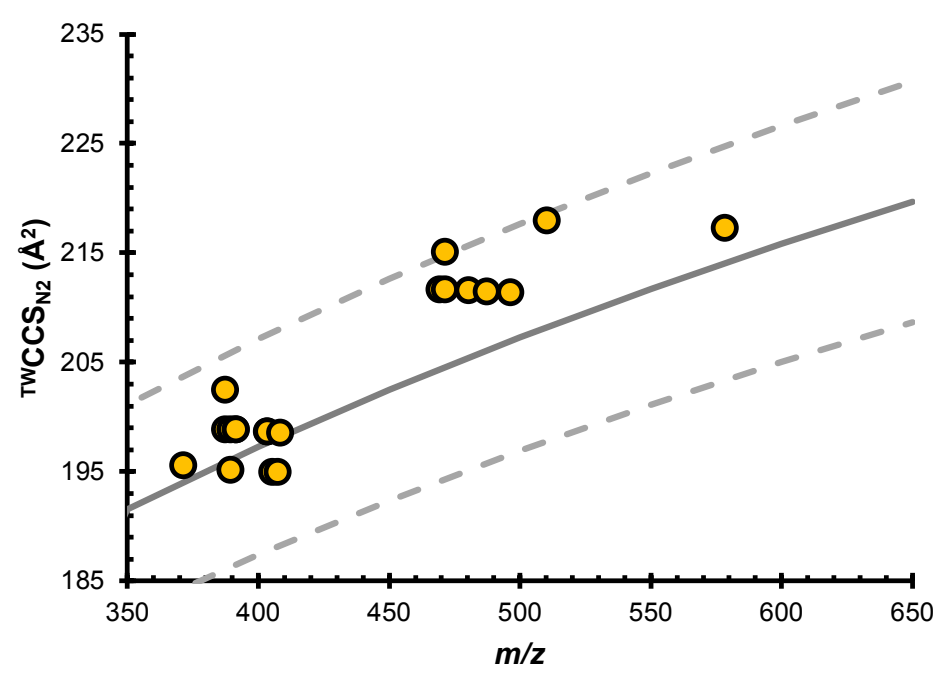

\begin{tabular}{|c|c|c|}
\hline Feature & $m / z$ & ${ }^{{ }^{T W}} \operatorname{CCS}_{\mathrm{N} 2}\left(\AA^{2}\right)$ \\
\hline 18.26_510.2526m/z & 510.25 & 218.0 \\
\hline $8.06578 .2464 \mathrm{~m} / \mathrm{z}$ & 578.25 & 217.3 \\
\hline 20.71_472.2495n & 471.24 & 215.1 \\
\hline 18.66_469.2261m/z & 469.23 & 211.7 \\
\hline 18.95_472.2506n & 471.24 & 211.7 \\
\hline 23.03_480.2791m/z & 480.28 & 211.6 \\
\hline 8.04_488.2438n & 487.24 & 211.5 \\
\hline $20.93 \_496.2734 \mathrm{~m} / \mathrm{z}$ & 496.27 & 211.4 \\
\hline $22.69 \_387.2546 \mathrm{~m} / \mathrm{z}$ & 387.25 & 202.5 \\
\hline $23.05 \_387.2537 \mathrm{~m} / \mathrm{z}$ & 387.25 & 198.9 \\
\hline 20.91_390.2770n & 389.27 & 198.9 \\
\hline 23.98_392.2943n & 391.29 & 198.9 \\
\hline 23.98_391.3341m/z & 391.33 & 198.9 \\
\hline $15.72 \_403.2489 \mathrm{~m} / \mathrm{z}$ & 403.25 & 198.7 \\
\hline 13.02_403.2983m/z & 403.30 & 198.7 \\
\hline $19.62 \_408.2839 n$ & 408.28 & 198.6 \\
\hline 24.13_371.2549m/z & 371.25 & 195.6 \\
\hline 23.29_389.2693m/z & 389.27 & 195.2 \\
\hline 14.52_405.2646m/z & 405.26 & 195.0 \\
\hline 16.61_407.3288m/z & 407.33 & 195.0 \\
\hline
\end{tabular}

Figure S4. Graph and associated data table showing previously unidentified metabolites and where they fall on the reported [M-H]- bile acid trendline. 
Supporting Information

\begin{tabular}{|c|c|c|c|c|c|c|c|c|c|}
\hline \multirow[b]{2}{*}{ Name } & \multirow{2}{*}{$\begin{array}{l}\text { Molecular } \\
\text { Formula }\end{array}$} & \multirow[b]{2}{*}{ MW (Da) } & \multicolumn{7}{|c|}{${ }^{\mathrm{DT}^{\top} \mathrm{CCS}_{\mathrm{N} 2}\left(\AA^{2}\right)}$} \\
\hline & & & $\begin{array}{c}\mathrm{MM}-2 \mathrm{SO}_{3}-2 \mathrm{H}_{2} \mathrm{O} \\
+\mathrm{H}]^{+} \\
\end{array}$ & \begin{tabular}{|c|}
$\begin{array}{c}{\left[\mathrm{M}-\mathrm{SO}_{3}-3 \mathrm{H}_{2} \mathrm{O}\right.} \\
+\mathrm{H}]^{+}\end{array}$ \\
\end{tabular} & 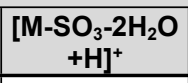 & $\begin{array}{c}{\left[\begin{array}{c}{\left[\mathrm{M}-\mathrm{SO}_{3}-\mathrm{H}_{2} \mathrm{O}\right.} \\
+\mathrm{H}]^{+}\end{array}\right.} \\
\end{array}$ & {$\left[\mathrm{M}-2 \mathrm{H}_{2} \mathrm{O}+\mathrm{H}\right]^{+}$} & {$\left[\mathrm{M}-\mathrm{H}_{2} \mathrm{O}+\mathrm{H}\right]^{+}$} & {$[\mathrm{M}+\mathrm{Na}]^{+}$} \\
\hline Dehydrolithocholic acid & $\mathrm{C} 24 \mathrm{H} 38 \mathrm{O} 3$ & 373.28 & -- & -- & -- & -- & -- & -- & $201.4 \pm 0.1$ \\
\hline Lithocholic acid & $\mathrm{C} 24 \mathrm{H} 40 \mathrm{O} 3$ & 376.3 & -- & -- & -- & -- & -- & $191.6 \pm 0.3$ & $214.8 \pm 0.3$ \\
\hline 3,6-diketocholic acid & $\mathrm{C} 24 \mathrm{H} 36 \mathrm{O} 4$ & 388.26 & -- & -- & -- & -- & $184.7 \pm 0.3$ & $188.8 \pm 0.3$ & $218.8 \pm 0.3$ \\
\hline 3,7-dioxy-5ß-choanoic acid & $\mathrm{C} 24 \mathrm{H} 36 \mathrm{O} 4$ & 388.26 & -- & -- & -- & -- & $182.2 \pm 0.1$ & $185.0 \pm 0.2$ & $201.8 \pm 0.3$ \\
\hline Dehydrodeoxycholic acid & $\mathrm{C} 24 \mathrm{H} 36 \mathrm{O} 4$ & 388.26 & -- & -- & -- & -- & $182.2 \pm 0.2$ & $188.0 \pm 0.2$ & $197.5 \pm 0.1$ \\
\hline 3-oxo-12a-cholic acid & $\mathrm{C} 24 \mathrm{H} 38 \mathrm{O} 4$ & 390.28 & -- & -- & -- & -- & $182.1 \pm 0.3$ & $191.5 \pm 0.3$ & $192.1 \pm 0.2$ \\
\hline Apocholic acid & $\mathrm{C} 24 \mathrm{H} 38 \mathrm{O} 4$ & 390.28 & -- & -- & -- & -- & $184.5 \pm 0.3$ & $190.7 \pm 0.2$ & $202.4 \pm 0.2$ \\
\hline Chenodeoxycholic acid & $\mathrm{C} 24 \mathrm{H} 40 \mathrm{O} 4$ & 392.29 & -- & -- & -- & -- & $186.9 \pm 0.2$ & -- & $202.8 \pm 0.2$ \\
\hline Hyodeoxycholic acid & $\mathrm{C} 24 \mathrm{H} 40 \mathrm{O} 4$ & 392.29 & -- & -- & -- & -- & $186.8 \pm 0.1$ & -- & $219.1 \pm 0.2$ \\
\hline Murideoxycholic acid & $\mathrm{C} 24 \mathrm{H} 40 \mathrm{O} 4$ & 392.29 & -- & -- & -- & -- & -- & -- & $222.5 \pm 0.3$ \\
\hline Ursodeoxycholic acid & $\mathrm{C} 24 \mathrm{H} 40 \mathrm{O} 4$ & 392.29 & -- & -- & -- & -- & $186.8 \pm 0.1$ & -- & -- \\
\hline $7 \alpha, 12 \alpha$, dihydroxy- $5 \beta$-cholanic acid & $\mathrm{C} 24 \mathrm{H} 40 \mathrm{O} 4$ & 392.3 & -- & -- & -- & -- & $186.4 \pm 0.1$ & -- & $203.7 \pm 0.2$ \\
\hline 3,7,12-tri-betacholanic acid & $\mathrm{C} 24 \mathrm{H} 34 \mathrm{O} 5$ & 402.24 & -- & -- & -- & -- & $185.3 \pm 0.3$ & $188.5 \pm 0.2$ & $201.2 \pm 0.2$ \\
\hline 7,12-dioxolithocholic acid & $\mathrm{C} 24 \mathrm{H} 36 \mathrm{O} 5$ & 404.26 & -- & -- & -- & -- & $185.5 \pm 0.2$ & $194.9 \pm 0.3$ & $204.8 \pm 0.1$ \\
\hline 12-dehydrocholic acid & $\mathrm{C} 24 \mathrm{H} 38 \mathrm{O} 5$ & 406.27 & -- & -- & -- & -- & $186.6 \pm 0.0$ & $191.2 \pm 0.3$ & $204.6 \pm 0.3$ \\
\hline 3-oxocholic acid & $\mathrm{C} 24 \mathrm{H} 38 \mathrm{O} 5$ & 406.27 & -- & -- & -- & -- & $182.5 \pm 0.2$ & $186.2 \pm 0.2$ & $201.5 \pm 0.1$ \\
\hline $3 \beta, 7 \alpha$, dihydroxy-5$\beta$-cholanic acid & $\mathrm{C} 24 \mathrm{H} 39 \mathrm{DO} 4$ & 406.27 & -- & -- & -- & -- & $186.5 \pm 0.0$ & -- & $210.0 \pm 0.2$ \\
\hline 7-ketodeoxycholic acid & $\mathrm{C} 24 \mathrm{H} 38 \mathrm{O} 5$ & 406.27 & -- & -- & -- & -- & $186.6 \pm 0.2$ & $204.8 \pm 0.3$ & $204.5 \pm 0.2$ \\
\hline Hyocholic acid & $\mathrm{C} 24 \mathrm{H} 40 \mathrm{O} 5$ & 408.29 & -- & -- & -- & -- & $203.3 \pm 0.1$ & 199.6 & $218.4 \pm 0.2$ \\
\hline a-Muricholic acid & $\mathrm{C} 24 \mathrm{H} 40 \mathrm{O} 5$ & 408.29 & -- & -- & -- & -- & $187.4 \pm 0.2$ & -- & $222.8 \pm 0.1$ \\
\hline$\beta$-Muricholic acid & $\mathrm{C} 24 \mathrm{H} 40 \mathrm{O} 5$ & 408.29 & -- & -- & -- & -- & $192.4 \pm 0.1$ & $201.3 \pm 0.2$ & $222.60 \pm .1$ \\
\hline Glycolithocholic acid & $\mathrm{C} 26 \mathrm{H} 43 \mathrm{NO} 4$ & 433.32 & -- & -- & -- & -- & -- & $209.2 \pm 0.2$ & $211.6 \pm 0.2$ \\
\hline Glycodeoxycholic acid & $\mathrm{C} 26 \mathrm{H} 43 \mathrm{NO} 5$ & 449.31 & -- & -- & -- & -- & $204.2 \pm 0.2$ & $207.2 \pm 0.2$ & $207.6 \pm 0.4$ \\
\hline Glycoursodeoxycholic acid & $\mathrm{C} 26 \mathrm{H} 43 \mathrm{NO} 5$ & 449.31 & -- & -- & -- & -- & $202.8 \pm 0.5$ & -- & $211.1 \pm 0.3$ \\
\hline Lithocholic acid 3-sulfate & $\mathrm{C} 24 \mathrm{H} 40 \mathrm{O} 6 \mathrm{~S}$ & 456.25 & -- & -- & -- & $191.6 \pm 0.1$ & -- & -- & -- \\
\hline Glycocholic acid & $\mathrm{C} 26 \mathrm{H} 43 \mathrm{NO} 6$ & 465.31 & -- & -- & -- & -- & $196.9 \pm 0.2$ & $197.4 \pm 0.2$ & $202.8 \pm 0.1$ \\
\hline Chenodeoxycholic acid 3-sulfate & $\mathrm{C} 24 \mathrm{H} 40 \mathrm{O} 7 \mathrm{~S}$ & 472.25 & -- & -- & $186.7 \pm 0.2$ & -- & -- & -- & -- \\
\hline Chenodeoxycholic acid 7-sulfate & $\mathrm{C} 24 \mathrm{H} 40 \mathrm{O} 7 \mathrm{~S}$ & 472.25 & -- & -- & $186.6 \pm 0.1$ & -- & -- & -- & -- \\
\hline Deoxycholic acid 3-sulfate & $\mathrm{C} 24 \mathrm{H} 40 \mathrm{O} 7 \mathrm{~S}$ & 472.25 & -- & -- & $187.4 \pm 0.2$ & -- & -- & -- & -- \\
\hline Deoxycholic acid 17 -sulfate & $\mathrm{C} 24 \mathrm{H} 40 \mathrm{O} 7 \mathrm{~S}$ & 472.25 & -- & -- & $187.4 \pm 0.2$ & -- & -- & -- & -- \\
\hline Taurolithocholic acid & $\mathrm{C} 26 \mathrm{H} 45 \mathrm{NO} 5 \mathrm{~S}$ & 483.3 & -- & -- & -- & -- & -- & $219.3 \pm 0.3$ & $214.2 \pm 0.3$ \\
\hline
\end{tabular}


Supporting Information

\begin{tabular}{|c|c|c|c|c|c|c|c|c|c|}
\hline \multirow[b]{2}{*}{ Name } & \multirow{2}{*}{$\begin{array}{l}\text { Molecular } \\
\text { Formula }\end{array}$} & \multirow[b]{2}{*}{ MW (Da) } & \multicolumn{7}{|c|}{${ }^{\mathrm{DT}} \mathrm{CCS}_{\mathrm{N} 2}\left(\AA^{2}\right)$} \\
\hline & & & $\begin{array}{c}{\left[\mathrm{M}-2 \mathrm{SO}_{3}-2 \mathrm{H}_{2} \mathrm{O}\right.} \\
\left.+\mathrm{H}^{+}\right]^{+}\end{array}$ & $\begin{array}{c}{\left[\mathrm{M}-\mathrm{SO}_{3}-3 \mathrm{H}_{2} \mathrm{O}\right.} \\
+\mathrm{H}]^{+}\end{array}$ & $\begin{array}{c}{\left[\mathrm{M}-\mathrm{SO}_{3}-2 \mathrm{H}_{2} \mathrm{O}\right.} \\
+\mathrm{H}]^{+}\end{array}$ & $\begin{array}{c}{\left[\mathrm{M}-\mathrm{SO}_{3}-\mathrm{H}_{2} \mathrm{O}\right.} \\
+\mathrm{H}]^{+}\end{array}$ & {$\left[\mathrm{M}-2 \mathrm{H}_{2} \mathrm{O}+\mathrm{H}\right]^{+}$} & {$\left[\mathrm{M}-\mathrm{H}_{2} \mathrm{O}+\mathrm{H}\right]^{+}$} & {$[\mathrm{M}+\mathrm{Na}]^{+}$} \\
\hline Cholic acid 3-sulfate & $\mathrm{C} 24 \mathrm{H} 40 \mathrm{O} 8 \mathrm{~S}$ & 488.244 & -- & $186.1 \pm 0.2$ & $189.6 \pm 0.2$ & -- & -- & -- & -- \\
\hline Cholic acid 12-sulfate & $\mathrm{C} 24 \mathrm{H} 40 \mathrm{O} 8 \mathrm{~S}$ & 488.24 & -- & $186.4 \pm 0.1$ & $190.3 \pm 0.1$ & -- & -- & -- & -- \\
\hline Taurochenodeoxycholic acid & $\mathrm{C} 26 \mathrm{H} 45 \mathrm{NO} 6 \mathrm{~S}$ & 499.3 & -- & -- & -- & -- & $218.7 \pm 0.1$ & -- & -- \\
\hline Taurohyodeoxycholic acid & $\mathrm{C} 26 \mathrm{H} 45 \mathrm{NO} 6 \mathrm{~S}$ & 499.3 & -- & -- & -- & -- & $204.5 \pm 0.2$ & $204.5 \pm 0.1$ & $206.7 \pm 0.2$ \\
\hline Tauroursodeoxycholic acid & $\mathrm{C} 26 \mathrm{H} 45 \mathrm{NO} 6 \mathrm{~S}$ & 499.3 & -- & -- & -- & -- & $202.6 \pm 0.2$ & -- & $216.4 \pm 0.3$ \\
\hline Glycolithocholic acid 3-sulfate & $\mathrm{C} 26 \mathrm{H} 43 \mathrm{NO} 7 \mathrm{~S}$ & 513.28 & -- & -- & -- & $209.0 \pm 0.1$ & -- & -- & -- \\
\hline Taurocholic acid & $\mathrm{C} 26 \mathrm{H} 45 \mathrm{NO} 7 \mathrm{~S}$ & 515.29 & -- & -- & -- & -- & $200.7 \pm 0.1$ & $204.6 \pm 0.2$ & $211.1 \pm 0.2$ \\
\hline Taurohyocholic acid & $\mathrm{C} 26 \mathrm{H} 45 \mathrm{NO} 7 \mathrm{~S}$ & 515.29 & -- & -- & -- & -- & $206.7 \pm 0.3$ & $206.2 \pm 0.1$ & $213.3 \pm 0.2$ \\
\hline Tauro- $\alpha-$ muricholic acid & $\mathrm{C} 26 \mathrm{H} 45 \mathrm{NO} 7 \mathrm{~S}$ & 515.29 & -- & -- & -- & -- & $201.2 \pm 0.3$ & -- & $213.1 \pm 0.4$ \\
\hline Tauro- $\beta$-muricholic acid & $\mathrm{C} 26 \mathrm{H} 45 \mathrm{NO} 7 \mathrm{~S}$ & 515.29 & -- & -- & -- & -- & $203.3 \pm 0.3$ & $203.2 \pm 0.2$ & $218.3 \pm 0.3$ \\
\hline Tauro- $\omega$-muricholic acid & $\mathrm{C} 26 \mathrm{H} 45 \mathrm{NO} 7 \mathrm{~S}$ & 515.29 & -- & -- & -- & -- & $201.9 \pm 0.2$ & $209.2 \pm 0.2$ & $221.9 \pm 3.1$ \\
\hline Glycochenodeoxycholic acid 3-sulfate & $\mathrm{C} 26 \mathrm{H} 43 \mathrm{NO} 8 \mathrm{~S}$ & 529.27 & -- & -- & $201.9 \pm 0.3$ & $203.6 \pm 0.3$ & -- & -- & -- \\
\hline Glycodeoxycholic acid 12-sulfate & $\mathrm{C} 26 \mathrm{H} 43 \mathrm{NO} 8 \mathrm{~S}$ & 529.27 & -- & -- & $204.5 \pm 0.5$ & $202.8 \pm 1.2$ & -- & -- & -- \\
\hline Glycocholic acid 3-sulfate & C26H43NO9S & 545.27 & -- & $196.6 \pm 0.2$ & $200.6 \pm 0.2$ & $211.4 \pm 0.2$ & -- & -- & -- \\
\hline Glycocholic acid 12-sulfate & C26H43NO9S & 545.27 & -- & $196.9 \pm 0.1$ & $204.5 \pm 0.2$ & -- & -- & -- & $207.8 \pm 0.2$ \\
\hline Chenodeoxycholic acid, disulfate & $\mathrm{C} 24 \mathrm{H} 40 \mathrm{O} 10 \mathrm{~S} 2$ & 552.21 & $186.9 \pm 0.1$ & -- & -- & -- & -- & -- & -- \\
\hline Taurolithocholic acid 3-sulfate & $\mathrm{C} 26 \mathrm{H} 45 \mathrm{NO} 8 \mathrm{~S} 2$ & 563.26 & -- & -- & -- & $219.4 \pm 0.1$ & -- & -- & $219.5 \pm 0.2$ \\
\hline Taurochenodeoxycholic acid 3-sulfate & $\mathrm{C} 26 \mathrm{H} 45 \mathrm{NO9S} 2$ & 579.25 & -- & -- & $202.5 \pm 0.3$ & -- & -- & -- & -- \\
\hline Glycochenodeoxycholic acid disulfate & $\mathrm{C} 26 \mathrm{H} 43 \mathrm{NO} 11 \mathrm{~S} 2$ & 609.23 & $206.5 \pm 1.1$ & -- & -- & -- & -- & -- & -- \\
\hline
\end{tabular}

Table S1. Bile acid collision cross section values measured via drift tube ion mobility with nitrogen $\left({ }^{\mathrm{DT}} \mathrm{CCS}_{\mathrm{N}_{2}}\right)$ in positive ionization mode in this work. Standard deviations for ${ }^{\mathrm{DT} C C S_{\mathrm{N}_{2}}}$ values are reported from three replicate measurements $(\mathrm{n}=3)$ performed on three separate days. Entries are sorted by molecular weight (MW). 
Supporting Information

\begin{tabular}{|c|c|c|c|c|c|c|c|c|c|}
\hline \multirow[b]{2}{*}{ Name } & \multirow{2}{*}{$\begin{array}{l}\text { Molecular } \\
\text { Formula }\end{array}$} & \multirow[b]{2}{*}{ MW (Da) } & \multicolumn{7}{|c|}{${ }^{\mathrm{DT}} \operatorname{CCS}_{\mathrm{N} 2}\left(\AA^{2}\right)$} \\
\hline & & & $\begin{array}{c}{\left[\mathrm{M}-\mathrm{SO}_{3}-\mathrm{H}_{2} \mathrm{O}\right.} \\
-\mathrm{H}] \\
\end{array}$ & {$\left[\mathrm{M}^{-\mathrm{SO}_{3}-\mathrm{H}}\right]^{-}$} & $\begin{array}{c}{\left[\mathrm{M}-\mathrm{SO}_{3}-\mathrm{H}_{2} \mathrm{O}\right.} \\
+\mathrm{Cl}]]^{-}\end{array}$ & $\begin{array}{c}{\left[\mathrm{M}_{-} \mathrm{SO}_{3}-\mathrm{H}_{2} \mathrm{O}\right.} \\
+\mathrm{HCOO}]\end{array}$ & {$\left[\mathrm{M}-\mathrm{SO}_{3}+\mathrm{Cl}\right]$} & {$\left[\mathrm{M}-\mathrm{H}_{2} \mathrm{O}-\mathrm{H}\right]^{-}$} & {$[\mathrm{M}-\mathrm{H}]^{-}$} \\
\hline Dehydrolithocholic acid & $\mathrm{C} 24 \mathrm{H} 38 \mathrm{O} 3$ & 374.28 & -- & -- & -- & -- & -- & -- & $205.5 \pm 0.2$ \\
\hline Lithocholic acid & $\mathrm{C} 24 \mathrm{H} 40 \mathrm{O} 3$ & 376.30 & -- & -- & -- & -- & -- & -- & $198.9 \pm 0.6$ \\
\hline 3,6-diketocholic acid & $\mathrm{C} 24 \mathrm{H} 36 \mathrm{O} 4$ & 388.26 & -- & -- & -- & -- & -- & -- & $200.9 \pm 0.4$ \\
\hline 3,7-dioxy-5ß-choanoic acid & $\mathrm{C} 24 \mathrm{H} 36 \mathrm{O} 4$ & 388.26 & -- & -- & -- & -- & -- & -- & -- \\
\hline Dehydrodeoxycholic acid & $\mathrm{C} 24 \mathrm{H} 36 \mathrm{O} 4$ & 388.26 & -- & -- & -- & -- & -- & -- & $190.7 \pm 1.6$ \\
\hline 3-oxo-12a-cholic acid & $\mathrm{C} 24 \mathrm{H} 38 \mathrm{O} 4$ & 390.28 & -- & -- & -- & -- & - & $193.2 \pm 0.0$ & $200.8 \pm 0.1$ \\
\hline Apocholic acid & $\mathrm{C} 24 \mathrm{H} 38 \mathrm{O} 4$ & 390.28 & -- & -- & -- & -- & - & $197.3 \pm 0.5$ & $189.1 \pm 0.5$ \\
\hline $7 \alpha, 12 \alpha$, dihydroxy- $5 \beta$-cholanic acid & $\mathrm{C} 24 \mathrm{H} 40 \mathrm{O} 4$ & 392.29 & -- & -- & -- & -- & -- & $194.9 \pm 0.3$ & $200.6 \pm 0.2$ \\
\hline Chenodeoxycholic acid & $\mathrm{C} 24 \mathrm{H} 40 \mathrm{O} 4$ & 392.29 & -- & -- & -- & -- & -- & -- & $202.3 \pm 0.2$ \\
\hline Hyodeoxycholic acid & $\mathrm{C} 24 \mathrm{H} 40 \mathrm{O} 4$ & 392.29 & -- & -- & -- & -- & -- & -- & $199.2 \pm 0.5$ \\
\hline Murideoxycholic acid & $\mathrm{C} 24 \mathrm{H} 40 \mathrm{O} 4$ & 392.29 & -- & -- & -- & -- & -- & -- & $200.3 \pm 0.5$ \\
\hline Ursodeoxycholic acid & $\mathrm{C} 24 \mathrm{H} 40 \mathrm{O} 4$ & 392.29 & -- & -- & -- & -- & -- & -- & $198.3 \pm 0.4$ \\
\hline $3 \beta, 7 \alpha$, dihydroxy-5$\beta$-cholanic acid & $\mathrm{C} 24 \mathrm{H} 39 \mathrm{DO} 4$ & 393.30 & -- & -- & -- & -- & -- & -- & $202.7 \pm 0.1$ \\
\hline 3,7,12-tri-betacholanic acid & $\mathrm{C} 24 \mathrm{H} 34 \mathrm{O} 5$ & 402.24 & -- & -- & -- & -- & -- & $191.9 \pm 0.4$ & $192.4 \pm 0.1$ \\
\hline 7,12-dioxolithocholic acid & $\mathrm{C} 24 \mathrm{H} 36 \mathrm{O} 5$ & 404.26 & -- & -- & -- & -- & -- & $194.8 \pm 0.7$ & $190.3 \pm 0.4$ \\
\hline 7-ketodeoxycholic acid & $\mathrm{C} 24 \mathrm{H} 38 \mathrm{O} 5$ & 406.27 & -- & -- & -- & -- & -- & -- & $204.2 \pm 0.1$ \\
\hline Cholic acid & $\mathrm{C} 24 \mathrm{H} 40 \mathrm{O} 5$ & 408.29 & -- & -- & -- & -- & -- & -- & $202.9 \pm 0.3$ \\
\hline Hyocholic acid & $\mathrm{C} 24 \mathrm{H} 40 \mathrm{O} 5$ & 408.29 & -- & -- & -- & -- & -- & $196.4 \pm 0.2$ & $190.1 \pm 0.3$ \\
\hline a-Muricholic acid & $\mathrm{C} 24 \mathrm{H} 40 \mathrm{O} 5$ & 408.29 & -- & -- & -- & - & -- & $200.8 \pm 0.3$ & $192.4 \pm 0.2$ \\
\hline$\beta$-Muricholic acid & $\mathrm{C} 24 \mathrm{H} 40 \mathrm{O} 5$ & 408.29 & -- & -- & -- & -- & -- & $205.7 \pm 0.3$ & $190.8 \pm 0.1$ \\
\hline Glycolithocholic acid & $\mathrm{C} 26 \mathrm{H} 43 \mathrm{NO} 4$ & 433.32 & -- & -- & -- & -- & -- & -- & $199.5 \pm 0.2$ \\
\hline Glycodeoxycholic acid & $\mathrm{C} 26 \mathrm{H} 43 \mathrm{NO} 5$ & 449.31 & -- & -- & -- & - & -- & - & $199.9 \pm 0.2$ \\
\hline Glycoursodeoxycholic acid & $\mathrm{C} 26 \mathrm{H} 43 \mathrm{NO} 5$ & 449.31 & -- & -- & -- & -- & -- & -- & $201.1 \pm 0.1$ \\
\hline Lithocholic acid 3-sulfate & $\mathrm{C} 24 \mathrm{H} 40 \mathrm{O} 6 \mathrm{~S}$ & 456.25 & -- & -- & -- & -- & -- & -- & $196.6 \pm 0.6$ \\
\hline Glycocholic acid & $\mathrm{C} 26 \mathrm{H} 43 \mathrm{NO} 6$ & 465.31 & -- & -- & -- & -- & - & - & $202.2 \pm 0.1$ \\
\hline Chenodeoxycholic acid 3-sulfate & $\mathrm{C} 24 \mathrm{H} 40 \mathrm{O} 7 \mathrm{~S}$ & 472.25 & $199.1 \pm 0.5$ & $202.7 \pm 0.2$ & -- & -- & -- & -- & $194.9 \pm 0.0$ \\
\hline Chenodeoxycholic acid 7-sulfate & $\mathrm{C} 24 \mathrm{H} 40 \mathrm{O} 7 \mathrm{~S}$ & 472.25 & $197.6 \pm 0.7$ & -- & -- & -- & -- & -- & $202.4 \pm 2.0$ \\
\hline Deoxycholic acid 3-sulfate & $\mathrm{C} 24 \mathrm{H} 40 \mathrm{O} 7 \mathrm{~S}$ & 472.25 & $198.1 \pm 0.1$ & $202.2 \pm 0.2$ & -- & -- & -- & $202.5 \pm 0.2$ & $196.3 \pm 0.6$ \\
\hline Deoxycholic acid 17-sulfate & $\mathrm{C} 24 \mathrm{H} 40 \mathrm{O} 7 \mathrm{~S}$ & 472.25 & -- & $201.9 \pm 0.1$ & -- & -- & - & -- & $206.1 \pm 0.1$ \\
\hline Taurolithocholic acid & $\mathrm{C} 26 \mathrm{H} 45 \mathrm{NO} 5 \mathrm{~S}$ & 483.30 & -- & -- & -- & -- & -- & -- & $206.4 \pm 0.1$ \\
\hline Cholic acid 3-sulfate & $\mathrm{C} 24 \mathrm{H} 40 \mathrm{O} 8 \mathrm{~S}$ & 488.24 & $199.3 \pm 0.1$ & $202.6 \pm 0.2$ & $204.3 \pm 0.2$ & $210.3 \pm 0.2$ & $203.6 \pm 0.1$ & $212.0 \pm 0.1$ & $192.4 \pm 0.2$ \\
\hline
\end{tabular}


Supporting Information

\begin{tabular}{|c|c|c|c|c|c|c|c|c|c|}
\hline \multirow[b]{2}{*}{ Name } & \multirow{2}{*}{$\begin{array}{l}\text { Molecular } \\
\text { Formula }\end{array}$} & \multirow[b]{2}{*}{ MW (Da) } & \multicolumn{7}{|c|}{${ }^{\mathrm{DT}} \operatorname{CCS}_{\mathrm{N} 2}\left(\AA^{2}\right)$} \\
\hline & & & $\begin{array}{c}\mathrm{M}-\mathrm{SO}_{3}-\mathrm{H}_{2} \mathrm{O} \\
-\mathrm{H}] \\
\end{array}$ & {$\left[\mathrm{M}-\mathrm{SO}_{3}-\mathrm{H}\right]^{-}$} & $\begin{array}{c}\begin{array}{c}\mathrm{M}^{-\mathrm{SO}_{3}}-\mathrm{H}_{2} \mathrm{O} \\
+\mathrm{Cl}]\end{array} \\
\end{array}$ & $\begin{array}{c}\text { [M-SO }_{3}-\mathrm{H}_{2} \mathrm{O} \\
+\mathrm{HCOO}] \\
\end{array}$ & {$\left[\mathrm{M}-\mathrm{SO}_{3}+\mathrm{Cl}\right]^{-}$} & {$\left[\mathrm{M}-\mathrm{H}_{2} \mathrm{O}-\mathrm{H}\right]^{-}$} & {$[\mathrm{M}-\mathrm{H}]^{-}$} \\
\hline Cholic acid 12 -sulfate & $\mathrm{C} 24 \mathrm{H} 40 \mathrm{O} 8 \mathrm{~S}$ & 488.24 & -- & -- & -- & -- & -- & -- & $207.7 \pm 0.2$ \\
\hline Taurochenodeoxycholic acid & $\mathrm{C} 26 \mathrm{H} 45 \mathrm{NO} 6 \mathrm{~S}$ & 499.30 & -- & -- & -- & -- & -- & -- & $207.2 \pm 0.2$ \\
\hline Taurohyodeoxycholic acid & $\mathrm{C} 26 \mathrm{H} 45 \mathrm{NO} 6 \mathrm{~S}$ & 499.30 & -- & -- & -- & -- & -- & -- & $206.3 \pm 0.2$ \\
\hline Tauroursodeoxycholic acid & $\mathrm{C} 26 \mathrm{H} 45 \mathrm{NO} 6 \mathrm{~S}$ & 499.30 & -- & -- & -- & -- & -- & -- & $207.6 \pm 0.1$ \\
\hline Glycolithocholic acid 3-sulfate & $\mathrm{C} 26 \mathrm{H} 43 \mathrm{NO}$ - & 513.28 & $200.1 \pm 0.4$ & $199.0 \pm 0.2$ & -- & -- & -- & - & $215.6 \pm 0.2$ \\
\hline Taurocholic acid & $\mathrm{C} 26 \mathrm{H} 45 \mathrm{NO} 7 \mathrm{~S}$ & 515.29 & -- & -- & -- & -- & -- & -- & $207.6 \pm 0.2$ \\
\hline Taurohyocholic acid & $\mathrm{C} 26 \mathrm{H} 45 \mathrm{NO} 7 \mathrm{~S}$ & 515.29 & -- & -- & -- & -- & -- & $210.2 \pm 0.4$ & $208.4 \pm 0.2$ \\
\hline Tauro-a-muricholic acid & $\mathrm{C} 26 \mathrm{H} 45 \mathrm{NO} 7 \mathrm{~S}$ & 515.29 & -- & -- & -- & -- & -- & -- & $209.4 \pm 0.1$ \\
\hline Tauro- $\beta$-muricholic acid & $\mathrm{C} 26 \mathrm{H} 45 \mathrm{NO}$ - & 515.29 & -- & -- & -- & -- & -- & $211.9 \pm 0.3$ & $209.5 \pm 0.2$ \\
\hline Tauro- $\omega$-muricholic acid & $\mathrm{C} 26 \mathrm{H} 45 \mathrm{NO} 7 \mathrm{~S}$ & 515.29 & -- & -- & -- & -- & -- & -- & $209.3 \pm 0.6$ \\
\hline Glycochenodeoxycholic acid 3-sulfate & $\mathrm{C} 26 \mathrm{H} 43 \mathrm{NO} 8 \mathrm{~S}$ & 529.27 & $200.3 \pm 0.1$ & $200.3 \pm 0.1$ & -- & $204.7 \pm 0.1$ & -- & $214.8 \pm 0.1$ & $215.2 \pm 0.1$ \\
\hline Glycodeoxycholic acid 12-sulfate & $\mathrm{C} 26 \mathrm{H} 43 \mathrm{NO} 8 \mathrm{~S}$ & 529.27 & -- & $199.5 \pm 0.0$ & -- & -- & -- & -- & $213.0 \pm 0.2$ \\
\hline Glycocholic acid 3-sulfate & $\mathrm{C} 26 \mathrm{H} 43 \mathrm{NO} 09 \mathrm{~S}$ & 545.27 & $200.2 \pm 0.1$ & $201.9 \pm 0.1$ & -- & -- & -- & $215.0 \pm 0.1$ & $215.8 \pm 0.1$ \\
\hline Glycocholic acid 12-sulfate & $\mathrm{C} 26 \mathrm{H} 43 \mathrm{~N} 09 \mathrm{~S}$ & 545.27 & -- & -- & -- & -- & -- & -- & $212.6 \pm 0.1$ \\
\hline Chenodeoxycholic acid, disulfate & $\mathrm{C} 24 \mathrm{H} 40 \mathrm{O} 10 \mathrm{~S} 2$ & 552.21 & $203.1 \pm 0.0$ & $203.9 \pm 0.0$ & -- & $219.1 \pm 0.1$ & -- & - & $207.6 \pm 0.2$ \\
\hline Taurolithocholic acid 3-sulfate & $\mathrm{C} 26 \mathrm{H} 45 \mathrm{NO} 8 \mathrm{~S} 2$ & 563.26 & $206.9 \pm 0.0$ & $206.3 \pm 0.1$ & -- & -- & -- & -- & $217.1 \pm 0.1$ \\
\hline Glycochenodeoxycholic acid disulfate & $\mathrm{C} 26 \mathrm{H} 43 \mathrm{NO} 11 \mathrm{~S} 2$ & 609.23 & $214.4 \pm 0.0$ & $212.8 \pm 0.1$ & -- & -- & -- & -- & $223.7 \pm 0.0$ \\
\hline
\end{tabular}

Table S2. Bile acid collision cross section values measured via drift tube ion mobility with nitrogen $\left({ }^{\mathrm{DT}} \mathrm{CCS}_{\mathrm{N}_{2}}\right)$ in negative ionization mode in this work, including ion forms with $\mathrm{m} / \mathrm{z}$ values less than the neutral mass. Additional ion forms observed in negative ionization mode are reported in Table $\mathrm{S}_{3}$. Standard deviations for ${ }^{\mathrm{DT}} \mathrm{CCS}_{\mathrm{N}_{2}}$ values are reported from three replicate measurements $(\mathrm{n}=3)$ performed on three separate days. Entries are sorted by molecular weight $(\mathrm{MW})$. 
Supporting Information

\begin{tabular}{|c|c|c|c|c|c|c|c|c|c|c|}
\hline \multirow[b]{2}{*}{ Name } & \multirow{2}{*}{$\begin{array}{l}\text { Molecular } \\
\text { Formula }\end{array}$} & \multirow[b]{2}{*}{ MW (Da) } & \multicolumn{8}{|c|}{${ }^{\mathrm{DT}} \mathrm{CCS}_{\mathrm{N} 2}\left(\AA^{2}\right)$} \\
\hline & & & {$[\mathrm{M}+\mathrm{Na}-2 \mathrm{H}]^{-}$} & $\begin{array}{c}{[\mathrm{M}-\mathrm{H} 2 \mathrm{O}} \\
+\mathrm{HCOO}^{-} \\
\end{array}$ & {$[\mathrm{M}+\mathrm{K}-2 \mathrm{H}]^{-}$} & {$\left[\mathrm{M}+\mathrm{HCOO}^{-}\right.$} & {$[\mathrm{M}+\mathrm{Na}-\mathrm{H}+\mathrm{Cl}]^{-}$} & $\begin{array}{l}{[\mathrm{M}+\mathrm{Na}-\mathrm{H}} \\
+\mathrm{HCOO}^{-} \\
\end{array}$ & {$[\mathrm{M}+\mathrm{K}-\mathrm{H}+\mathrm{Cl}]^{-}$} & $\begin{array}{r}{[\mathrm{M}+\mathrm{K}-\mathrm{H}} \\
+\mathrm{HCOO}] \\
\end{array}$ \\
\hline Dehydrolithocholic acid & $\mathrm{C} 24 \mathrm{H} 38 \mathrm{O} 3$ & 374.28 & $190.6 \pm 0.3$ & -- & -- & $201.1 \pm 0.1$ & $197.5 \pm 0.1$ & $200.6 \pm 0.1$ & -- & $203.2 \pm 0.2$ \\
\hline Lithocholic acid & $\mathrm{C} 24 \mathrm{H} 40 \mathrm{O} 3$ & 376.30 & -- & -- & -- & $198.8 \pm 0.2$ & $197.9 \pm 0.0$ & $201.6 \pm 0.1$ & -- & $204.1 \pm 0.2$ \\
\hline 3,6-diketocholic acid & $\mathrm{C} 24 \mathrm{H} 36 \mathrm{O} 4$ & 388.26 & $190.9 \pm 0.1$ & -- & $203.4 \pm 0.0$ & $199.6 \pm 0.1$ & $197.5 \pm 0.1$ & $200.6 \pm 0.1$ & -- & -- \\
\hline 3,7-dioxy-5 $\beta$-choanoic acid & $\mathrm{C} 24 \mathrm{H} 36 \mathrm{O} 4$ & 388.26 & $191.3 \pm 0.1$ & -- & -- & $202.2 \pm 0.1$ & $199.4 \pm 0.3$ & $205.2 \pm 2.8$ & $203.1 \pm 0.2$ & $205.7 \pm 0.4$ \\
\hline Dehydrodeoxycholic acid & $\mathrm{C} 24 \mathrm{H} 36 \mathrm{O} 4$ & 388.26 & -- & -- & -- & $206.2 \pm 0.3$ & $200.1 \pm 0.0$ & $208.4 \pm 0.1$ & -- & $205.2 \pm 0.0$ \\
\hline 3-oxo-12a-cholic acid & $\mathrm{C} 24 \mathrm{H} 38 \mathrm{O} 4$ & 390.28 & $200.9 \pm 0.9$ & - & -- & $201.2 \pm 1.8$ & $199.0 \pm 0.1$ & $202.5 \pm 0.1$ & $202.4 \pm 0.1$ & -- \\
\hline Apocholic acid & $\mathrm{C} 24 \mathrm{H} 38 \mathrm{O} 4$ & 390.28 & -- & -- & -- & $201.4 \pm 0.1$ & $198.8 \pm 0.1$ & $202.4 \pm 0.1$ & $201.8 \pm 0.3$ & -- \\
\hline 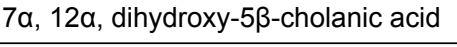 & $\mathrm{C} 24 \mathrm{H} 40 \mathrm{O} 4$ & 392.29 & $199.5 \pm 0.3$ & -- & -- & $202.2 \pm 0.5$ & $199.1 \pm 0.2$ & $202.4 \pm 0.1$ & $202.1 \pm 0.2$ & -- \\
\hline Chenodeoxycholic acid & $\mathrm{C} 24 \mathrm{H} 40 \mathrm{O} 4$ & 392.29 & -- & -- & -- & $201.9 \pm 0.1$ & $199.2 \pm 0.0$ & $205.8 \pm 0.1$ & -- & $208.5 \pm 0.1$ \\
\hline Hyodeoxycholic acid & $\mathrm{C} 24 \mathrm{H} 40 \mathrm{O} 4$ & 392.29 & $194.8 \pm 0.6$ & -- & -- & $200.4 \pm 0.3$ & $199.5 \pm 0.2$ & $203.6 \pm 0.2$ & $203.1 \pm 1.5$ & $206.5 \pm 0.3$ \\
\hline Murideoxycholic acid & $\mathrm{C} 24 \mathrm{H} 40 \mathrm{O} 4$ & 392.29 & -- & -- & -- & $200.6 \pm 0.5$ & $200.0 \pm 0.1$ & $204.0 \pm 0.1$ & $203.7 \pm 0.1$ & $206.1 \pm 0.9$ \\
\hline Ursodeoxycholic acid & $\mathrm{C} 24 \mathrm{H} 40 \mathrm{O} 4$ & 392.29 & -- & -- & -- & $200.2 \pm 0.2$ & $199.4 \pm 0.2$ & $203.2 \pm 0.1$ & -- & $205.7 \pm 0.2$ \\
\hline $3 \beta, 7 \alpha$, dihydroxy-5 $\beta$-cholanic acid & $\mathrm{C} 24 \mathrm{H} 39 \mathrm{DO} 4$ & 393.30 & -- & -- & -- & $204.1 \pm 0.2$ & $202.4 \pm 0.0$ & $206.3 \pm 0.1$ & -- & $209.1 \pm 0.2$ \\
\hline 3,7,12-tri-betacholanic acid & $\mathrm{C} 24 \mathrm{H} 34 \mathrm{O} 5$ & 402.24 & $192.8 \pm 0.3$ & -- & $207.2 \pm 1.2$ & $206.1 \pm 0.1$ & $202.7 \pm 0.1$ & $206.9 \pm 0.1$ & -- & $208.7 \pm 0.1$ \\
\hline 7,12-dioxolithocholic acid & $\mathrm{C} 24 \mathrm{H} 36 \mathrm{O} 5$ & 404.26 & $203.2 \pm 0.2$ & -- & -- & $201.3 \pm 0.3$ & $200.4 \pm 0.1$ & $206.1 \pm 0.1$ & -- & -- \\
\hline 7-ketodeoxycholic acid & $\mathrm{C} 24 \mathrm{H} 38 \mathrm{O} 5$ & 406.27 & -- & -- & $203.2 \pm 0.6$ & $202.1 \pm 0.4$ & $200.4 \pm 0.1$ & $204.1 \pm 0.1$ & -- & $205.6 \pm 0.7$ \\
\hline Cholic acid & $\mathrm{C} 24 \mathrm{H} 40 \mathrm{O} 5$ & 408.29 & -- & -- & -- & $203.0 \pm 0.2$ & $199.8 \pm 0.1$ & $204.5 \pm 2.1$ & -- & $205.7 \pm 1.0$ \\
\hline Hyocholic acid & $\mathrm{C} 24 \mathrm{H} 40 \mathrm{O} 5$ & 408.29 & $197.0 \pm 0.6$ & $197.8 \pm 0.3$ & -- & $202.5 \pm 0.2$ & $200.1 \pm 0.2$ & $206.8 \pm 0.2$ & -- & $209.2 \pm 0.3$ \\
\hline a-Muricholic acid & $\mathrm{C} 24 \mathrm{H} 40 \mathrm{O} 5$ & 408.29 & $194.5 \pm 0.2$ & -- & -- & $203.7 \pm 0.2$ & $201.0 \pm 0.2$ & $207.8 \pm 0.2$ & -- & $209.8 \pm 0.1$ \\
\hline$\beta$-Muricholic acid & $\mathrm{C} 24 \mathrm{H} 40 \mathrm{O} 5$ & 408.29 & $202.8 \pm 0.3$ & -- & -- & $201.9 \pm 0.3$ & $201.3 \pm 0.1$ & $206.0 \pm 0.2$ & -- & $209.2 \pm 0.3$ \\
\hline Glycolithocholic acid & $\mathrm{C} 26 \mathrm{H} 43 \mathrm{NO} 4$ & 433.32 & -- & -- & -- & -- & $211.8 \pm 0.2$ & $214.5 \pm 0.2$ & -- & $216.9 \pm 0.2$ \\
\hline Glycodeoxycholic acid & $\mathrm{C} 26 \mathrm{H} 43 \mathrm{NO} 5$ & 449.31 & -- & -- & -- & -- & $212.3 \pm 0.2$ & $214.0 \pm 0.2$ & -- & $214.2 \pm 1.4$ \\
\hline Glycoursodeoxycholic acid & $\mathrm{C} 26 \mathrm{H} 43 \mathrm{NO} 5$ & 449.31 & -- & -- & -- & -- & $213.5 \pm 0.4$ & $215.5 \pm 0.1$ & -- & $218.0 \pm 0.1$ \\
\hline Lithocholic acid 3-sulfate & $\mathrm{C} 24 \mathrm{H} 40 \mathrm{O} 6 \mathrm{~S}$ & 456.25 & $202.1 \pm 0.1$ & $208.9 \pm 0.3$ & $205.5 \pm 0.1$ & -- & -- & -- & -- & -- \\
\hline Glycocholic acid & $\mathrm{C} 26 \mathrm{H} 43 \mathrm{NO} 6$ & 465.31 & $204.8 \pm 0.4$ & -- & -- & -- & $210.7 \pm 0.1$ & $212.2 \pm 0.2$ & -- & $214.2 \pm 0.4$ \\
\hline Dehydrolithocholic acid & $\mathrm{C} 24 \mathrm{H} 38 \mathrm{O} 4$ & 445.47 & $190.6 \pm 0.4$ & -- & -- & $201.1 \pm 0.2$ & $197.5 \pm 0.2$ & $200.6 \pm 0.2$ & $214.3 \pm 1.1$ & $215.4 \pm 0.1$ \\
\hline Lithocholic acid & $\mathrm{C} 24 \mathrm{H} 40 \mathrm{O} 4$ & 448.50 & -- & - & -- & $198.8 \pm 0.3$ & $197.9 \pm 0.1$ & $201.6 \pm 0.2$ & -- & $217.6 \pm 0.2$ \\
\hline 3,6-diketocholic acid & $\mathrm{C} 24 \mathrm{H} 36 \mathrm{O} 4$ & 451.54 & $190.9 \pm 0.2$ & -- & $203.4 \pm 0.1$ & $199.6 \pm 0.2$ & $197.5 \pm 0.2$ & $200.6 \pm 0.2$ & $212.0 \pm 1.2$ & $212.4 \pm 0.5$ \\
\hline 3,7-dioxy-5ß-choanoic acid & $\mathrm{C} 24 \mathrm{H} 36 \mathrm{O} 4$ & 454.57 & $191.3 \pm 0.2$ & -- & -- & $202.2 \pm 0.2$ & $199.4 \pm 0.4$ & $205.2 \pm 2.9$ & -- & -- \\
\hline Dehydrodeoxycholic acid & $\mathrm{C} 24 \mathrm{H} 36 \mathrm{O} 4$ & 457.61 & -- & -- & -- & $206.2 \pm 0.4$ & $200.1 \pm 0.1$ & $208.4 \pm 0.2$ & -- & $221.4 \pm 0.3$ \\
\hline 3-oxo-12a-cholic acid & $\mathrm{C} 24 \mathrm{H} 38 \mathrm{O} 4$ & 460.64 & $200.9 \pm 0.10$ & -- & -- & $201.2 \pm 1.9$ & $199.0 \pm 0.2$ & $202.5 \pm 0.2$ & $213.4 \pm 0.3$ & $212.7 \pm 0.2$ \\
\hline
\end{tabular}


Supporting Information

\begin{tabular}{|c|c|c|c|c|c|c|c|c|c|c|}
\hline \multirow[b]{2}{*}{ Name } & \multirow{2}{*}{$\begin{array}{l}\text { Molecular } \\
\text { Formula }\end{array}$} & \multirow[b]{2}{*}{ MW (Da) } & \multicolumn{8}{|c|}{${ }^{\mathrm{DT}} \mathrm{CCS}_{\mathrm{N} 2}\left(\AA^{2}\right)$} \\
\hline & & & {$[\mathrm{M}+\mathrm{Na}-2 \mathrm{H}]^{-}$} & $\begin{array}{c}\text { [M-H2O } \\
+\mathrm{HCOO}] \\
\end{array}$ & {$[\mathrm{M}+\mathrm{K}-2 \mathrm{H}]^{-}$} & {$[\mathrm{M}+\mathrm{HCOO}]^{-}$} & {$[\mathrm{M}+\mathrm{Na}-\mathrm{H}+\mathrm{Cl}]^{-}$} & $\begin{array}{l}\text { [M+Na-H } \\
+\mathrm{HCOO}^{-} \\
\end{array}$ & {$[\mathrm{M}+\mathrm{K}-\mathrm{H}+\mathrm{Cl}]^{-}$} & $\begin{array}{r}\text { [M+K-H } \\
+\mathrm{HCOO}] \\
\end{array}$ \\
\hline Apocholic acid & $\mathrm{C} 24 \mathrm{H} 38 \mathrm{O} 4$ & 463.68 & -- & -- & -- & $201.4 \pm 0.2$ & $198.8 \pm 0.2$ & $202.4 \pm 0.1$ & -- & $217.7 \pm 0.2$ \\
\hline $7 \alpha, 12 \alpha$, dihydroxy-5 $\beta$-cholanic acid & $\mathrm{C} 24 \mathrm{H} 40 \mathrm{O} 4$ & 466.72 & $199.5 \pm 0.4$ & -- & -- & $202.2 \pm 0.6$ & $199.1 \pm 0.3$ & $202.4 \pm 0.1$ & -- & $223.3 \pm 0.3$ \\
\hline Chenodeoxycholic acid & $\mathrm{C} 24 \mathrm{H} 40 \mathrm{O} 4$ & 469.75 & -- & -- & -- & $201.9 \pm 0.2$ & $199.2 \pm 0.1$ & $205.8 \pm 0.2$ & -- & $224.5 \pm 0.1$ \\
\hline Hyodeoxycholic acid & $\mathrm{C} 24 \mathrm{H} 40 \mathrm{O} 4$ & 472.79 & $194.8 \pm 0.7$ & -- & -- & $200.4 \pm 0.4$ & $199.5 \pm 0.3$ & $203.6 \pm 0.3$ & -- & $223.9 \pm 0.2$ \\
\hline Murideoxycholic acid & $\mathrm{C} 24 \mathrm{H} 40 \mathrm{O} 4$ & 475.82 & -- & -- & -- & $200.6 \pm 0.6$ & $200.0 \pm 0.2$ & $204.0 \pm 0.2$ & -- & -- \\
\hline Ursodeoxycholic acid & $\mathrm{C} 24 \mathrm{H} 40 \mathrm{O} 4$ & 478.86 & -- & -- & -- & $200.2 \pm 0.3$ & $199.4 \pm 0.3$ & $203.2 \pm 0.2$ & -- & -- \\
\hline $3 \beta, 7 \alpha$, dihydroxy- $5 \beta$-cholanic acid & C24H39DO5 & 481.89 & -- & -- & -- & $204.1 \pm 0.3$ & $202.4 \pm 0.1$ & $206.3 \pm 0.2$ & -- & $224.6 \pm 1.1$ \\
\hline 3,7,12-tri-betacholanic acid & $\mathrm{C} 24 \mathrm{H} 34 \mathrm{O} 6$ & 484.93 & $192.8 \pm 0.4$ & -- & $207.2 \pm 1.3$ & $206.1 \pm 0.2$ & $202.7 \pm 0.2$ & $206.9 \pm 0.2$ & -- & $225.0 \pm 1.0$ \\
\hline 7,12-dioxolithocholic acid & $\mathrm{C} 24 \mathrm{H} 36 \mathrm{O} 6$ & 487.96 & $203.2 \pm 0.3$ & -- & -- & $201.3 \pm 0.4$ & $200.4 \pm 0.1$ & $206.1 \pm 0.2$ & -- & -- \\
\hline 7-ketodeoxycholic acid & $\mathrm{C} 24 \mathrm{H} 38 \mathrm{O} 6$ & 491.00 & -- & -- & $203.2 \pm 0.7$ & $202.1 \pm 0.5$ & $200.4 \pm 0.1$ & $204.1 \pm 0.2$ & -- & -- \\
\hline Glycochenodeoxycholic acid 3-sulfate & $\mathrm{C} 26 \mathrm{H} 43 \mathrm{NO} 0 \mathrm{~S}$ & 529.27 & $216.5 \pm 0.1$ & $219.5 \pm 0.1$ & $217.8 \pm 0.8$ & -- & -- & -- & $223.3 \pm 0.2$ & $224.2 \pm 0.2$ \\
\hline Glycodeoxycholic acid 12-sulfate & $\mathrm{C} 26 \mathrm{H} 43 \mathrm{NO} 8 \mathrm{~S}$ & 529.27 & $219.0 \pm 0.2$ & $220.3 \pm 1.3$ & $215.5 \pm 0.3$ & -- & -- & -- & -- & -- \\
\hline Glycocholic acid 3-sulfate & $\mathrm{C} 26 \mathrm{H} 43 \mathrm{NO} O \mathrm{~S}$ & 545.27 & $217.1 \pm 0.1$ & -- & -- & -- & -- & -- & - & -- \\
\hline Glycocholic acid 12-sulfate & $\mathrm{C} 26 \mathrm{H} 43 \mathrm{NO} 09 \mathrm{~S}$ & 545.27 & $212.7 \pm 0.1$ & -- & $215.3 \pm 0.1$ & -- & -- & -- & -- & -- \\
\hline Chenodeoxycholic acid, disulfate & $\mathrm{C} 24 \mathrm{H} 40 \mathrm{O} 10 \mathrm{~S} 2$ & 552.21 & $228.1 \pm 0.1$ & -- & -- & - & - & - & - & -- \\
\hline Taurolithocholic acid 3-sulfate & $\mathrm{C} 26 \mathrm{H} 45 \mathrm{NO} 8 \mathrm{~S} 2$ & 563.26 & $222.8 \pm 0.1$ & -- & $225.2 \pm 0.0$ & -- & -- & -- & -- & -- \\
\hline Glycochenodeoxycholic acid disulfate & $\mathrm{C} 26 \mathrm{H} 43 \mathrm{NO} 11 \mathrm{~S} 2$ & 609.23 & $223.6 \pm 0.0$ & -- & -- & -- & - & -- & -- & -- \\
\hline
\end{tabular}

Table $\mathrm{S}_{3}$. Bile acid collision cross section values measured via drift tube ion mobility with nitrogen $\left({ }^{\mathrm{DT}} \mathrm{CCS}_{\mathrm{N}_{2}}\right)$ in negative ionization mode in this work, including ion forms with $\mathrm{m} / \mathrm{z}$ values greater than the neutral mass. Additional ion forms observed in negative ionization mode are reported in Table S2. Standard deviations for ${ }^{\text {DT }} \mathrm{CCS}_{\mathrm{N}_{2}}$ values are reported from three replicate measurements $(\mathrm{n}=3)$ performed on three separate days. Entries are sorted by molecular weight $(\mathrm{MW})$. 